\section{An unusual case of}

\section{abdominal distension}

\section{with constipation}

\section{Pooja Popat, ${ }^{1}$ Helen Williams, ${ }^{2}$ Ian Wacogne, ${ }^{1}$ Ingo Jester, ${ }^{3}$ Silvia Planas, ${ }^{4}$ Mohamed A Elemraid ${ }^{1}$}

A previously healthy 3 -year-old boy was referred by his general practitioner to the paediatric outpatient clinic because of a 6-month history of moderate to severe constipation. It was associated with mild central abdominal pain and slight weight loss. He had been treated with Movicol, with minimal effect. There were no other symptoms or history of foreign travel. $\mathrm{He}$ appeared well, but had a non-tender distended abdomen with shifting dullness and fluid thrill suspicious of ascites. There was no bulging of abdominal flanks, pouting of the umbilicus or scrotal swelling. The rest of his systemic examination was normal.

He was admitted for investigations which included an abdominal radiograph (figure 1) and an ultrasound scan (USS) (figure 2) as well as normal liver function tests, a negative screen for viral hepatitis $\mathrm{B}$ and $\mathrm{C}$ and a negative Mantoux test. Following review of the imaging and discussion with the surgical team, he underwent MRI of his abdomen (figure 3).

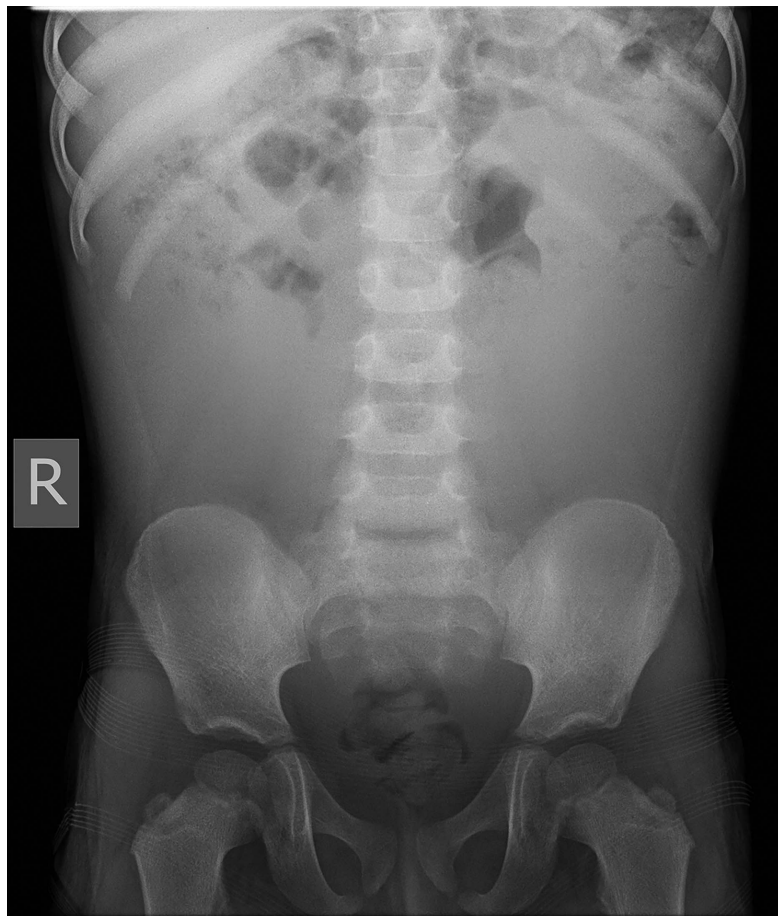

Figure 1 Plain abdominal radiograph.

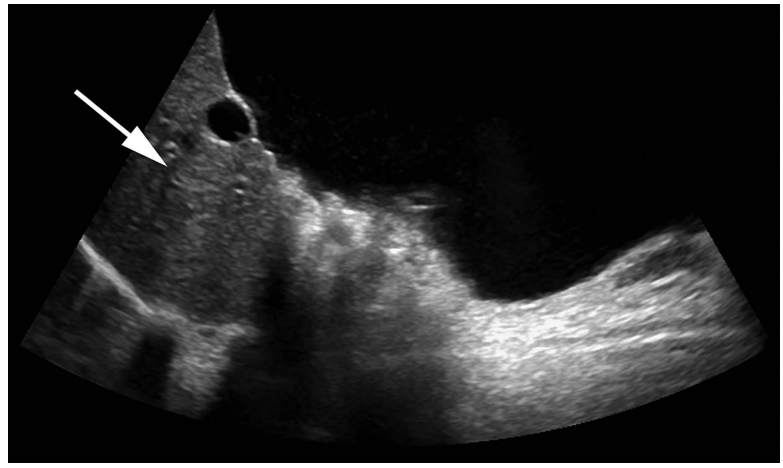

Figure 2 Right longitudinal image of ultrasound scan of abdomen including the liver.

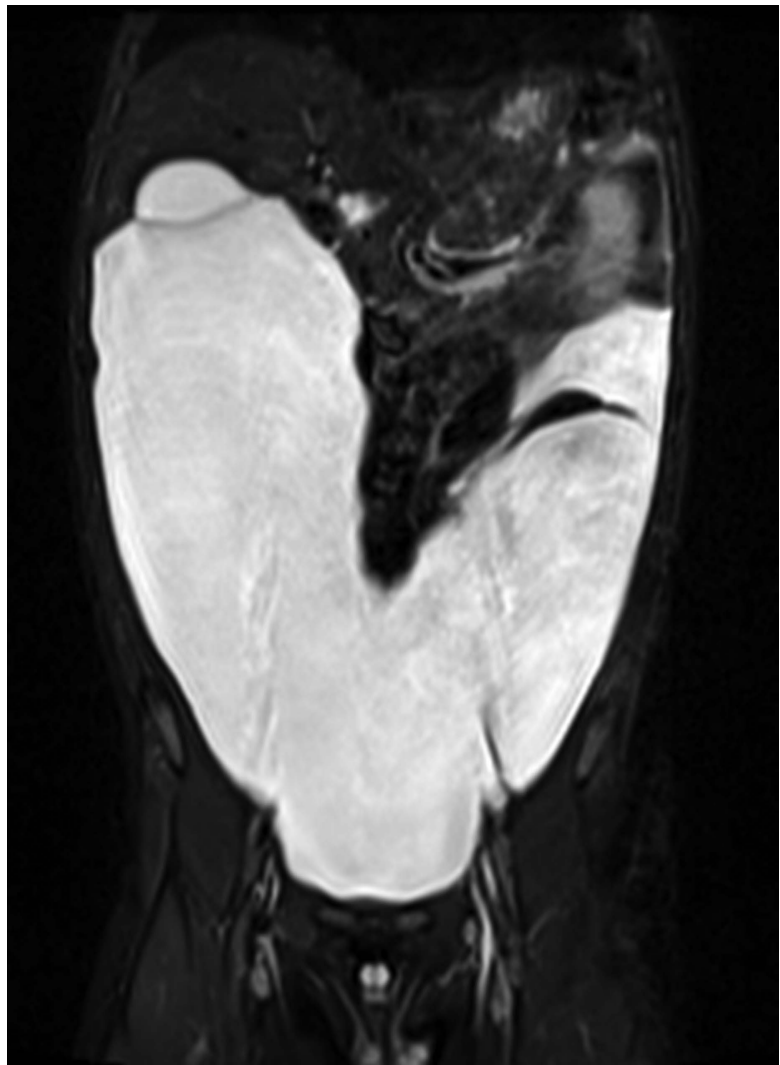

Figure 3 Coronal T2-weighted image with fat saturation of MRI scan of abdomen. Viscera and solid organs appear black and fluid appears white.

Test your knowledge

1. What does the abdominal radiograph show?

2. What abnormality was shown on the abdominal USS?

3. What does the MRI scan show?

4. What is the likely diagnosis?

5. What is the pathophysiology of this disease?

6. How else can this diagnosis present?

Answers to the questions are on page 168. 